\title{
sciendo
}

\author{
Current Issues in Pharmacy and Medical Sciences \\ Formerly ANNALES UNIVERSITATIS MARIAE CURIE-SKLODOWSKA, SECTIO DDD, PHARMACIA \\ journal homepage: http://www.curipms.umlub.pl/
}

\section{Acute liver injury in the course of lymphoplasmacytic lymphoma}

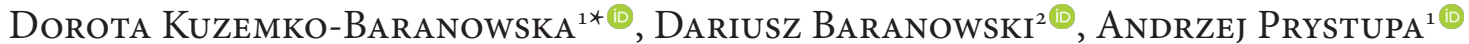

\author{
${ }^{1}$ Chair and Department of Internal Diseases, Medical University of Lublin Independent, Clinical Hospital No. 1 in Lublin, Staszica 16, \\ 20-081 Lublin, Poland \\ ${ }^{2}$ Chair and Department of Neurology, Medical University of Lublin, Independent Clinical Hospital No. 4 in Lublin, Jaczewskiego 8, \\ 20-954 Lublin, Poland
}

\section{ARTICLE INFO \\ Received 23 March 2018 \\ Accepted 19 April 2018}

\section{Keywords:}

lymphoma,

liver injury.

\begin{abstract}
Non-Hodgkin lymphomas are malignant neoplasms whose incidence rates increase each year. These also include neoplasms rare in the general population. The present case report described a patient with lymphoplasmacytic lymphoma (LPL) and rapid liver damage. In most cases, infiltration of the liver is rare in advanced stages of hematopoietic malignancies when hepatomegaly, cholestatic jaundice and organ failure are observed. The patient's history includes non-specific abdominal pain that was accompanied by general symptoms such as nocturnal hyperhidrosis, subfibrile temperature and fever, as well as weight loss. The above complaints aggravate with an increase in organ size. The laboratory findings initially demonstrated moderately elevated concentrations of transaminases. In our case, the baseline biochemical indices of liver function were found to be normal. During the next days of hospitalisation, the features of liver damage intensified and were accompanied by liver failure. The gold diagnostic standard is a biopsy of the bone marrow and the organ affected. Since the patient's condition deteriorated and liver failure developed, the diagnosis was established based on trephine biopsy of the bone marrow. Chemotherapy was implemented; despite the treatment applied, the patient's clinical condition did not improve. Two months after the onset of first symptoms the patient died.
\end{abstract}

Non-Hodgkin lymphomas (NHLs) are a group of systemic malignant diseases. NHL is the $6^{\text {th }}$ most common cancer in the Polish population, and constitutes about $2 \%$ of all malignancies. The annual incidence is estimated at 100000 inhabitants. The most alarming signal is an increasingly high number of newly affected patients, including those with rare B-cell lymphomas [1].

Lymphoplasmacytic lymphoma (LPL) is a neoplasm of the lymphatic system involving small B cells, lymphoplasmatic cells and plasmocytes. The cases in which LPL coexists with IgM monoclonal gammapathy (irrespective of the IgM concentration) and bone marrow involvement are called Waldenstrom macroglobulinaemia (WM).

The incidence of lymphoid cancers increases with age. The peak incidence for LPL-WM is observed in the $7^{\text {th }}$ decade of life, more commonly in males.

The affected lymphatic structures include the bone marrow (mainly LPL), lymph nodes and the spleen. The liver is rarely affected.

\footnotetext{
* Corresponding author

e-mail: dorota.kuzemko@gmail.com
}

Generally, the hepatic infiltrations occur in about $15-22 \%$ of all patients with hematopoietic neoplasms; neoplastic cells infiltrate small intrahepatic bile ducts resulting in hepatic vasoconstriction (secondary ischaemia), biliary necrosis or cholangitis. The longer the pathological process, the higher the number of degraded hepatocytes. The above process is initially accompanied by moderate increases in transaminase concentrations [2].

Abdominal pain associated with an increased organ size, cholestatic jaundice, progressive hepatitis or acute liver failure are rare, and predominantly in the advanced stages of disease.

The present case report describes a patient with rapid liver damage in the course of lymphoplasmacytic lymphoma.

A 54-year-old male patient in fair general condition was admitted to the Department of Internal Diseases due to abdominal pain increasingly severe for two months accompanied by nausea without vomiting. The patient, a non-smoker and moderate alcohol drinker, has been chronically treated for gout, prostatic hyperplasia and hypertriglyceridaemia. No allergic reactions to the drugs used were observed. The family history failed to reveal 
genetic, autoimmune or neoplastic diseases. The patient is a truck driver and delivers goods to the EU countries; the previous year he did not travel to remote world regions. His lifestyle is unhygienic (a fat-rich diet based on highly processed food).

The physical examination revealed normal skin and mucosa colour, no enlarged lymph nodes; resting ECG showed a regular heart rate of about $70 / \mathrm{min}$ and arterial pressure of $135 / 80 \mathrm{mmHg}$. On auscultation - normal alveolar sound above the lung fields, the distended abdomen, slightly tender in the right subcostal region; the peritoneal symptoms were negative. On palpation, the enlarged liver, its inferior border was palpable about $2 \mathrm{~cm}$ in the right midclavicular line below the costal arch.

On admission, the basic additional examinations were carried out . The biochemical indices of liver function were normal, except for elevated GGTP (502 IU/1, ref. 10-71 IU/1). The urine analysis disclosed albuminuria and haematuria. Abdominal ultrasound showed the enlargement of the liver $(150 \mathrm{~mm} \times 45 \mathrm{~mm})$ and prostate $(48 \mathrm{~m} \times 56 \mathrm{~mm})$.

Symptomatic treatment was applied, drotaverine and metamizole were administered. After treatment, the symptoms partially subsided.

Gastroscopy demonstrated the features of oesophagitis and gastritis, pyloric erosion and hiatus hernia. The material collected for histopathological examinations was positive for Helicobacter pylori.

The immunological tests did not reveal anti-mitochondrial antibodies (AMAs) nor anti-nuclear, anti-cytoplasmatic, anti-tissue glutaminase $\mathrm{IgA}$ and $\mathrm{IgG}$, anti-endomysial $\operatorname{IgA}$ and $\operatorname{IgG}$ and anti-Yersinia enterocolitica antibodies.

During the next hospitalisation days, the patient's condition deteriorated. On day 12, yellowish discolouration of the skin and mucosa was observed (total bilirubin concentration was $4.62 \mathrm{mg} / \mathrm{dl}$ ). The patient reported intensified abdominal pain. The laboratory tests disclosed an increase in biochemical indices of liver function: AST - $72 \mathrm{IU} / 1$ (ref. 5-50 IU/l), ALT - 64.1 IU/1 (ref. 5-45 IU/1), ALP - 469 IU/1 (ref. 10-119 IU/1), GGTP - 975 IU/1 (10-71 IU/1). The indices of liver efficiency were slightly changed, i.e. prolongation of prothrombin time and INR 1.53 (ref. 0.86-1.3); the concentration of albumin was reduced to $3.1 \mathrm{~g} / \mathrm{dl}$ (ref. 3.5-5.2 g/dl), Moreover, mild normocytic anaemia was noticed. According to the 4-degree scale of clinical severity of hepatic encephalopathy, the patient score was 1 . The patient developed acute liver damage without features of liver failure.

$\mathrm{HBV}$ and HCV infections were excluded, the history of CMV and EBV infections were confirmed.

The follow-up abdominal ultrasound demonstrated liver enlargement to $220 \mathrm{~mm}$ in the mid-clavicular line; the remaining structures were comparable to those observed in the previous examination. Abdominal CT with contrast and MR cholangiography revealed enlargement of the liver $(220 \times 225 \mathrm{~mm}$ in the frontal plane $)$, spleen $(144 \times 96 \times 60 \mathrm{~mm})$, numerous mesenteric lymph nodes (up to $16 \times 11 \mathrm{~mm}$ ) and periaortic lymph nodes (up to $17 \times 11 \mathrm{~mm}$ ).

The symptomatic treatment included ornithine aspartate, omeprazole and ursodeoxycholic acid; pain was managed with tramadol.
During the next days in the Department, jaundice became more severe (total bilirubin - $8.61 \mathrm{mg} / \mathrm{dl}$ (ref. 0,2-1,1 mg/dl), direct bilirubin $7.8 \mathrm{mg} / \mathrm{dl}$ (ref. 0,0-0,2 mg/dl) and ascites progressed. The follow-up laboratory tests demonstrated significantly elevated biochemical indices of liver function - AST - 204 IU/1 (ref. 5-50 IU/1), ALT - 94.6 IU/1 ref. 5-45 IU/1), ALP - 567 IU/1 (ref. 10-119 IU/1), GGTP $1251 \mathrm{IU} / 1$ (ref. 10-71 IU/1). The indices of liver efficiency were abnormal, i.e. prolonged prothrombin time and INR 1.94 (ref. 0,86-1,3), concentration of albumin $-2.7 \mathrm{~g} / \mathrm{dl}$ (ref. 3,5-5,2 mg/dl). Abdominal pain was assessed by the patient as very strong. According to the 4-degree scale, clinical hepatic encephalopathy was scored 2 . The renal function did not deteriorate. The patient developed acute liver damage with simultaneous deterioration of efficiency.

Since ascitis increased, furosemide and spironolactone were instituted. Moreover, an infusion of $20 \%$ albumin was added. Due to extremely severe abdominal pain, oxycodone was administered.

Considering substantial changes in the patient's condition and increasing symptoms of liver failure of unknown causes, the patient was consulted oncologically and haematologically. The following neoplastic markers were determined: AFP, CA 19-9 and CEA - the results were within normal limits. The levels of immunoglobulins - IgA, IgG and IgM, were normal. Protein electrophoresis and immunofixation were performed. Fractions in protein electrophoresis were obtained: albumin 51.5\% (ref. 55.8-66.1\%), alpha $111.9 \%$ (ref. 2.9-4.9\%), alpha $216.7 \%$ (ref. 7.1-11.8\%), beta1 5.7\% (ref. 4.7-7.2\%), beta2 8.1\% (ref. 3.2-6.5\%), gamma $6.1 \%$ (ref. 11.1-18.8\%); A/G ratio: 1.06, T.P: $3.78 \mathrm{~g} / \mathrm{dl}$. After detecting the monoclonal protein by immunofixation, a kappa band unrelated to the heavy chains was obtained. Among the results, the high concentration of free Kappa chains was of interest - $1320 \mathrm{mg} / \mathrm{l}$ ( ref. 3.3-19.4 mg/l); the concentration of free Lambda chains was within normal limits $-7.76 \mathrm{mg} / \mathrm{l}$ ( ref. 5.71-26.3 mg/l). The patient underwent aspiration biopsy of the bone marrow and trephine biopsy. The myelogram showed atypical lymphocytes of lymphoplasmacytic morphology.

The trephine bioptate demonstrated an infiltrate of lymphocytes and lymphoplasmacytes (CD 45+, CD 20+, CD 3-, IgM + in single cells), constituting about $15 \%$ of bone marrow texture.

Moreover, a small oral mucosal fragment was biopsied from the maxillary alveolar process; polarised light microscopy did not reveal amyloid deposits.

Based on the clinical picture and the results of additional examinations, the patient was diagnosed with lymphoplasmacytic lymphoma and transferred to the Department of Hemato-Oncology for causal treatment.

Therapy with cyclophosphamide, doxorubicin, vincristine and dexamethasone was initiated. The treatment of symptoms of acute liver damage was continued. Despite the treatment applied, no significant clinical improvement was observed. The patient developed severe liver failure and pancytopenia. After the first cycle of chemotherapy, the patient did not used rituximab, because the patient died about 3 weeks later. 


\section{DISCUSSION}

In our case, the first evident manifestation of lymphoma was acute fulminant liver failure. Initially, the symptoms were not characteristic. The patient reported abdominal pain accompanied by nausea yet did not observe such general symptoms as nocturnal hyperhidrosis, fever, subfebrile temperature or unexplainable weight loss.

Imaging examinations demonstrated hepatosplenomegaly and enlarged lymph nodes in the abdominal cavity. On admission, the biochemical indices of liver function were within reference range limits (except for elevated GGTP); the indices of liver efficiency were slightly altered.

During the subsequent hospitalisation days, the patient's condition systematically deteriorated. Despite the hepatoprotective and symptomatic treatment applied, his clinical condition did not improve. Physical examination revealed yellowish discolouration of the skin and mucosa, as well as ascites. Moreover, hepatic encephalopathy was aggravated. Imaging examinations disclosed liver enlargement (baseline $190 \mathrm{~mm}$ in the mid-clavicular line; after 2 weeks $-220 \mathrm{~mm}$ ). The de Ritis ratio was $>1$. The biochemical indices of liver function pointed to acute liver damage; the development of liver failure was also observed.

In cases with symptoms of liver failure, the differential diagnosis should firstly exclude the most common causes of acute failure of this organ. In developing countries, acute liver failure is associated with viral hepatitis (mostly caused by HBV, less commonly by HEV). In Western Europe and the United States, drug-induced liver damage is predominantly involved (acetaminophen). Among the remaining causes, intoxications (toadstool toxins) and autoimmune processes (autoimmune hepatitis) should be mentioned $[3,4]$. Whenever the cause-effect relation between acute liver failure and the above aetiological factors has not been demonstrated and the clinical picture is suggestive of some hematopoietic disease, diagnostic procedures for haematological neoplasms should be initiated.

The gold standard for diagnosis of lymphoplasmacytic lymphoma is histopathology of the bone marrow (trephine biopsy) or specimens biopsied from the affected organ.

Additional examinations include immunoelectrophoresis and immunofixation of proteins to determine the presence and concentrations of an abnormal IgM antibody.

Moreover, cerebrospinal fluid (CSF) analysis, in some cases also head MRI, can prove useful. When the myocardium is involved, echocardiography should be performed.

In special cases, cytofluorometric, molecular and cytogenetic examinations are carried out [5-7].

A similar clinical course is observed in primary hepatic lymphoma (PHL). Although the incidence of PHL as a variant of non-Hodgkin lymphoma is $<1 \%$, PHL should be considered when other haematological causes of an episode of acute liver failure have been excluded.
In the case described, liver failure progressed rapidly without any evident cause and therefore the patient was consulted haematologically and oncologically. Considering hepatosplenomegaly and enlarged mesenteric, as well as periaortic lymph nodes observed on an abdominal CT scan, electrophoresis and immunofixation of proteins were additionally performed. The latter demonstrated elevated concentrations of free Kappa chains, which was of interest. Furthermore, bone marrow trephine biopsy was performed.

Due to the patient's severe condition and substantially impaired liver function, the liver was not biopsied and the diagnosis of lymphoplasmacytic lymphoma was established based on histopathological examinations of bone marrow cells. These showed an infiltrate with lymphoplasmacytic cells and atypical lymphocytes. The decision was made to initiate chemotherapy in the Department of Hemato-Oncology setting.

The first-line therapy includes cladribine, fludarabin and chlorambucil; steroids were administered as adjuvants. In special cases, therapy combined with cyclophosphamide, doxorubicin, vincristine, prednisolone (CHOP) can be applied. Rituximab (a monoclonal antibody) has been approved for biological therapy.

In cases with slow courses of LPL/MW, the estimated survival is about 6 years, on average. When the course of disease is rapid, this survival time can be markedly shorter and the prognosis is bad [8]. In our case, the expected therapeutic effects were not achieved despite chemotherapy used. Two months after the occurrence of first symptoms, the patient died.

\section{SUMMARY}

Non-Hodgkin lymphomas are systemic neoplastic diseases, the incidence rates of which have systematically increased. In an increasingly high number of cases, their course is rapid and the treatment options are less numerous as the disease progresses. The diagnosis is often preceded by time-consuming and highly specialist procedures. Moreover, non-specific symptoms accompanying non-Hodgkin lymphoma can hinder its diagnosis. Liver infiltrates with lymphoma cells are rare and usually observed in advanced stages. When liver infiltrations are suspected, the gold diagnostic standard is organ biopsy. In our case, the result of trephine biopsy enabled to identify the aetiological factor of acute liver damage and to implement the causal treatment.

In everyday practice, this group of neoplasmas should be considered in the differential diagnosis of acute liver damage - as they are initially poorly symptomatic yet fulminant in advanced stages.

\section{ORCID iDs}

Dorota Kuzemko-Baranowska (Dhttps://orcid.org/0000-0002-6024-0714 Dariusz Baranowski Dhttps://orcid.org/0000-0002-4270-0422 Andrzej Prystupa Dhttps://orcid.org/0000-0003-4628-8911 


\section{REFERENCES}

1. Sawczuk-Chabin J, Centkowski P, Biliński P, Warzocha K Epidemiology of non-Hodgkin lymphomas. Acta Haematoa Poa. 2004;35 2):131-144.

2. Lettieri CJ, Berg BW. Clinical features of non-Hodgkins lymphoma presenting with acute liver failure: a report of five cases and review of published experience. Am J Gastroenterol. 2003;98(7):1641-6.

3. Sass D, Shakil A. Fulminant hepatic failure. Liver Transpl. 2005;11: 594-605.

4. Larson A: Diagnosis and management of acute liver failure. Curr Opin Gastroenterol. 2010; 26:214-221.

5. Pileri SA, Zinzani L, Sabattini E, et al. Lymphoplasmacytic lymphoma and marginal zone lymphoma: diagnostic challenges. Haematologica. 2005;90:148.
6. Xie Y, Vallangeon B, Liu X, Lagoo AS. Plasmacytic or lymphoplasmacytic infiltrate in lymph nodes: Diagnostic approach and differential considerations. Indian J Pathol Microbiol. 201c;59(4): 446-456. doi: 10.4103/0377-4929.191756.

7. Gertz MA. : Waldenström macroglobulinemia: 2017 update on diagnosis, risk stratification, and management. Am J Hematol. 201b;92(2):209-217. doi: 10.1002/ajh.24557.

8. Lech-Marańda E, Kalinka-Warzocha E, Warzocha K. Advances in the diagnosis and treatment of patients with non-Hodgkin's lymphomas. Hematologia. 2015;6(1):31-43. DOI: 10.5603/Hem.2015. 0013 